\title{
Simulation and experimental analysis of indoor localization systems
}

\begin{abstract}
Indoor localization systems, especially its accuracy, have become a major topic in recent research. Moreover, location-based services have been used in many mobile applications and wireless sensor networks. High accuracy and fast convergence are crucial requirements for a good localization system. However, the type of obtained received signal strength (RSS) data is vital to obtain high accuracy. In this paper, we perform a simulation and an experimental analysis for an indoor localization system. Moreover, 2D and 3D localization models are considered to investigate the localization error. The simulation is developed to provide an accurate RSS compared with an experimental testbed by considering the multiwall path loss model. Results demonstrate that the simulated RSS data are consistent with the experimental RSS data; that is, the simulated data almost have same distribution form as the experimental data. Therefore, the simulation can be used to develop high-accuracy localization systems.
\end{abstract}

Keyword: Bayesian network; Fingerprinting; Localization 